TAIWANESE JOURNAL OF MATHEMATICS

Vol. 8, No. 4, pp. 703-712, December 2004

This paper is available online at http://www.math.nthu.edu.tw/tjm/

\title{
MINIMAX INEQUALITIES IN THE SPACES WITHOUT LINEAR STRUCTURE
}

\author{
Lu Haishu and Zhang Jihui
}

\begin{abstract}
In this paper, we found a new result by relaxing the condition of [15, Corollary 2]. As its application, we have obtained some new minimax inequalities of Ky Fan and minimax theorems in the spaces without linear structure.
\end{abstract}

\section{Introduction And PReliminaries}

Since Ky Fan ([8]) generalized $K K M$ theorem, a number of applications have been found. Fan's theorem is now becoming a very versatile tool in nonlinear analysis, such as fixed point, variational inequalities (see [2-7]). Fan's theorem was used by many authors to prove fixed point and minimax theorems in topological vector spaces (see [3], [8]). Ha [1-2] has given the generalization of Fan's theorem and Fan's minimax inequalities. This paper has two purposes. First we obtain a new theorem by relaxing closed condition of sets of [15, Corollary 2] , and next, as its application, we obtain some minimax inequalities and minimax theorems in the spaces without linear structure.

To begin with we explain the notions of an $H$-space introduced by Horvath [9-11] and related concepts on $H$-spaces.

Let $X$ be a topological space and let $F(X)$ be the family of all nonempty finite subsets of $X$. Let $\left\{\Gamma_{A}\right\}$ be a family of nonempty contractible subsets of $X$ indexed by $A \in F(X)$ such that $\Gamma_{A} \subset \Gamma_{A^{\prime}}$, whenever $A \subset A^{\prime}$. The pair $\left(X,\left\{\Gamma_{A}\right\}\right)$ is called an $H$-space. Given an $H$-space $\left(X,\left\{\Gamma_{A}\right\}\right)$, a nonempty subset $D$ of $X$ is called

Received June 3, 2002; accepted July 18, 2003.

Communicated by Bor-Lnh Lin.

2000 Mathematics Subject Classification: 47H10, 49A29, 49J35.

Key words and phrases: Minimax theorems, $H$-spaces, $H$-convex, Upper (lower) semicontinuous, Set-valued mapping.

*This work supported by Natural Science Foundation of Jiangsu Province. 
$H$-convex if $\Gamma_{A} \subset D$ for each nonempty finite subset $A$ of $D$. For a nonempty $K$ of $X$, we define the $H$-convex hull of $K$, denote by $H$-co $K$, as

$$
H-c o K=\bigcap\{D \subset X: D \text { is } H \text {-convex and } D \supset K\} .
$$

Clearly, $H-c o K$ is $H$-convex and is the smallest $H$-convex containing $K$.

Let $\left(X,\left\{\Gamma_{A}\right\}\right)$ be an $H$-space, let $Y$ be a topological space, and let $f: X \times Y \rightarrow$ $R$ be a function. For each $y \in Y, f(x, y)$ is said to be $H$-quasiconvex (or $H$ quasiconcave ) on $X$ if the set $\{x \in X: f(x, y)<t\}$ (or $\{x \in X: f(x, y)>t\}$ ) is $H$-convex for all $t \in R$.

A topological space is called acyclic if all of its reduced $\breve{C} e c h$ homology groups over rationals vanish. In particular, any contractible space is acyclic, thus, any nonempty convex or star-shaped set is acyclic.

Now let $X, Y$ be two topological spaces. By a set-valued mapping $T$ defined on $X$ with values in $Y$, we mean that to each point $x \in X, T$ assigns an unique nonempty subset $T(x)$ of $Y$. T is called upper semicontinuous if for each open subset $G$ of $Y$, the set $\{x \in X: T(x) \subset G\}$ is open in $X$. It is easy to show (e.g., [14]) that if $Y$ is a compact Hausdorff and if $T(x)$ is closed for each $x$, then $T$ is upper semicontinuous if and only if the graph $\{(x, y) \in X \times Y: y \in T(x)\}$ of $T$ is closed in $X \times Y$.

\section{MAIN Results}

Our main result is the following Theorem 2.1. To prove it we need to cite a lemma in Tarafdar [12].

Lemma 2.1. Let $X$ be a compact topological space and let $\left(Y,\left\{\Gamma_{A}\right\}\right)$ be an $H$ space. Let $T: X \rightarrow 2^{Y}$ be a set-valued mapping and $T^{-1}(y)=\{x \in X: y \in T x\}$ for each $y \in Y$.

Suppose the following conditions are fulfilled:

(i) For each $x \in X, T(x)$ is a nonempty $H$-convex subset of $Y$,

(ii) $\left\{\operatorname{int} T^{-1}(y): y \in Y\right\}$ is an open covering of $X$.

Then there is a continuous selection $f: X \rightarrow Y$ of $T$ such that $f=g \varphi$, where $g: \Delta_{n} \rightarrow Y$ and $\varphi: X \rightarrow \Delta_{n}$ are continuous mappings, and $\Delta_{n}$ is the standard $n$-dimensional simplex for some positive $n$.

Theorem 2.1. Let $X$ be a Hausdorff topological space, let $\left(Y,\left\{\Gamma_{A}\right\}\right)$ be an $H$-space, and let $M, N$ be two subsets of $X \times Y$ with $M \subset N$. Suppose the following conditions are fulfilled: 
(i) For each $y \in Y$, there exists a closed subset $X_{y} \subset X$ such that the set $\{x \in X:(x, y) \in N\} \subset X_{y}$,

(ii) For each $x \in X$, the set $\{y \in Y:(x, y) \notin M\}$ is $H$-convex or empty.

Suppose also that there exists a subset $P$ of $M$ and a compact subset $K$ of $X$ such that $P$ is closed in $X \times Y$, and

(iii) For each $y \in Y$, the set $\{x \in K:(x, y) \in P\}$ is nonempty acyclic.

Then

$$
\bigcap_{y \in Y} X_{y} \bigcap K \neq \emptyset
$$

Proof. If the conclusion of Theorem 2.1 is false, that is, $\bigcap_{y \in Y} X_{y} \subset X \backslash K$, we prove that there exists $x_{0} \in K$ such that

$$
\left\{x_{0}\right\} \times Y \subset N,
$$

or else, for each $x \in K$, there exists $y_{0} \in Y$ such that $\left(x, y_{0}\right) \notin N$. Let

$$
S(x)=\{y \in Y:(x, y) \notin N\}, T(x)=\{y \in Y:(x, y) \notin M\} .
$$

Then $S, T: K \rightarrow 2^{Y}$ are two set-valued mappings such that for each $x \in K$, there exists a point $y \in S(x)$. By $S^{-1}(y)=\{x \in X:(x, y) \notin N\}$ and (i), $S^{-1}(y) \supset X \backslash X_{y}=V_{y}, V_{y}$ is an open set of $X$, and $\bigcup_{y \in Y} V_{y}=X \backslash \bigcap_{y \in Y} X_{y} \supset$ $K$. Therefore, $\left\{\operatorname{int} S^{-1}(y): y \in Y\right\}$ is an open covering of $K$. Consequently, $\left\{\operatorname{int}(H-c o S)^{-1}(y): y \in Y\right\}$ is an open covering of $K$, where the following mapping $H-\operatorname{coS}: K \rightarrow 2^{Y}$ is defined by

$$
H-\operatorname{coS}(x)=H-\operatorname{co}(S(x)) \quad \text { for each } x \in K .
$$

By Lemma 2.1, there exists a continuous mapping $f: K \rightarrow Y$ such that $f=g \Psi$, and

$$
f(x) \in H-\operatorname{coS}(x) \subset T(x)
$$

for all $x \in K$, where $\Psi: K \rightarrow \Delta_{n}, g: \Delta_{n} \rightarrow Y$ are continuous mappings and $\Delta_{n}$ is the standard $n$-simplex. Hence

$$
(x, f(x)) \notin M \quad \text { for all } x \in K .
$$

On the other hand, we define a set-valued mapping $G: Y \rightarrow 2^{K}$ by

$$
G(y)=\{x \in K:(x, y) \in P\} \quad \text { for all } y \in Y .
$$

By (iii), $G(y)$ is nonempty and acyclic for all $y \in Y$. Since $P$ is closed in $X \times Y$, each $G(y)$ is closed in $K$ and the graph of $G$ is closed in $Y \times K$; thus, $G$ is an upper 
semicontinuous set-valued mapping defined on $Y$. Consequently, so is the mapping $F: \Delta_{n} \rightarrow 2^{K}$ defined by $F(\mu)=G(g(\mu))$. By virtue of [13, Lemma 2.1], there exists a point $\bar{\mu} \in \Delta_{n}$ such that $\bar{\mu} \in \Psi(F(\bar{\mu}))=\Psi(G(g(\bar{\mu})))$, and so there is a point $\bar{x} \in G(g(\bar{\mu})) \subset K$ such that $\bar{\mu}=\Psi(\bar{x})$. Let $\bar{y}=g(\bar{\mu})$, then $\bar{y}=g(\Psi(\bar{x}))=f(\bar{x})$ and $\bar{x} \in G(\bar{y})$, i.e.,

$$
(\bar{x}, f(\bar{x}))=(\bar{x}, \bar{y}) \in P \subset M .
$$

This contradicts (2.2), hence, $(2.1)$ is true. But this contradicts $\bigcap_{y \in Y} X_{y} \subset X \backslash K$ again, therefore, we must have $\bigcap_{y \in Y} X_{y} \bigcap K \neq \emptyset$. This proves the theorem.

Remark 2.1. Theorem 2.1 improve and extend [16, Theorem 2] to a topological space and an $H$-space.

As an immediate consequence of Theorem 2.1, we obtain some new minimax theorems and some minimax inequalities in the spaces without linear structure.

Theorem 2.2. Let $X$ be a Hausdorff topological space. Let $\left(Y,\left\{\Gamma_{A}\right\}\right)$ be an $H$-space, and let $f, g, h: X \times Y \rightarrow R$ be functions. Let $\beta=\inf _{K \in \bar{K}} \sup _{y \in Y} \min _{x \in K} h(x, y)$ , where $\bar{K}=\{K \subset X: K$ is compact subset of $X\}$. Suppose the following conditions are fulfilled:

(i) $f(x, y) \leq g(x, y) \leq h(x, y)$ for all $(x, y) \in X \times Y$,

(ii) $f(x, y)$ is lower semicontinuous on $X$ for each $y \in Y$,

(iii) $g(x, y)$ is $H$-quasiconcave on $Y$ for each $x \in X$,

(iv) $h(x, y)$ is lower semicontinuous on $X \times Y$, and the set $\{x \in K: h(x, y)<t\}$ is acyclic or empty for each $t>\beta, K \in \bar{K}$ and $y \in Y$. Then

$$
\inf _{x \in X} \sup _{y \in Y} f(x, y) \leq \inf _{K \in \bar{K}} \sup _{y \in Y} \min _{x \in K} h(x, y) .
$$

If $X$ is compact, then

$$
\min _{x \in X} \sup _{y \in Y} f(x, y) \leq \sup _{y \in Y} \min _{x \in X} h(x, y) .
$$

Proof. We can assume that the right-hand side of $(2.3)$ is not $+\infty$. If the conclusion of Theorem 2.2 is false, then there is a real number $t$ such that

$$
\inf _{x \in X} \sup _{y \in Y} f(x, y)>t>\inf _{K \in \bar{K}} \sup _{y \in Y} \min _{x \in K} h(x, y) .
$$

Let $M=\{(x, y) \in X \times Y: g(x, y) \leq t\}$ and $P=\{(x, y) \in X \times Y: h(x, y) \leq t\}$. Then 
(a) For each $x \in X$, by (iii), the set $\{y \in Y:(x, y) \notin M\}$ is $H$-convex and satisfies (ii) of Theorem 2.1,

(b) For each $y \in Y$, by (i), $\{x \in X:(x, y) \in M\} \subset\{x \in X: f(x, y) \leq t\}=$ $X_{y}$, by (ii), $X_{y}$ is closed and satisfies (i) of Theorem 2.1. It is easy to verify that $P$ is closed in $X \times Y, P \subset M$,

(c) Let $K$ be a compact subset of $X$ such that

$$
t>\sup _{y \in Y} \min _{x \in K} h(x, y) .
$$

Then for any $y \in Y$, the set $\{x \in K: h(x, y) \leq t\}$ is nonempty and we know the set $\{x \in K: h(x, y) \leq t\}=\bigcap_{\epsilon>0}\{x \in K: h(x, y)<t+\epsilon\}$ is acyclic (this follows from the continuity of Cech homology. See, e.g., McClendon [17]). Thus, by Theorem 2.1,

$$
\bigcap_{y \in Y} X_{y} \bigcap K \neq \emptyset
$$

that is, there exists $x_{0} \in K$ such that

$$
f\left(x_{0}, y\right) \leq t \text { for all } y \in Y .
$$

Hence

$$
\inf _{x \in X} \sup _{y \in Y} f(x, y) \leq t .
$$

This contradicts the choice of $t$, therefore, (2.3) is proved.

We shall establish the following similarities of the proof of theorem 2.2.

Theorem 2.3. Let $f, g, h: X \times Y \rightarrow R$ be as in Theorem 2.2 and $X$ is compact. Then for each $\lambda \in R$, one of the following situations hold:

(i) There exists $x_{0} \in X$ such that $f\left(x_{0}, y\right) \leq \lambda$ for all $y \in Y$,

(ii) There exists $y_{0} \in Y$ such that $f\left(x, y_{0}\right)>\lambda$ for all $x \in X$.

Remark 2.2. Theorem 2.3 is the generalization of [16,Theorem 4].

The following three minimax theorems are obtained from Theorem 2.2 as special cases by taking $f=g, g=h, f=g=h$.

Corollary 2.1. Let $f, h: X \times Y \rightarrow R$ be two functions. Let $\beta=\inf _{K \in \bar{K}} \sup _{y \in Y} \min _{x \in K}$ $h(x, y)$, where $\bar{K}=\{K \subset X: K$ is compact subset of $X\}$. Suppose the following conditions are fulfilled:

(i) $f(x, y) \leq h(x, y)$ for all $(x, y) \in X \times Y$, 
(ii) $f(x, y)$ is lower semicontinuous on $X$ for each $y \in Y$, and $f(x, y)$ is $H$ quasiconcave on $Y$ for each $x \in X$,

(iii) $h(x, y)$ is lower semicontinuous on $X \times Y$ and the set $\{x \in K: h(x, y)<t\}$ is acyclic or empty for each $t>\beta, K \in \bar{K}$ and $y \in Y$. Then

$$
\inf _{x \in X} \sup _{y \in Y} f(x, y) \leq \inf _{K \in \bar{K}} \sup _{y \in Y} \min _{x \in K} h(x, y) .
$$

If $X$ is compact, then

$$
\min _{x \in X} \sup _{y \in Y} f(x, y) \leq \sup _{y \in Y} \min _{x \in X} h(x, y) .
$$

Corollary 2.2. Let $f, g: X \times Y \rightarrow R$ be two functions. Let $\beta=\inf _{K \in \bar{K}} \sup _{y \in Y} \min _{x \in K}$ $g(x, y)$, where $\bar{K}=\{K \subset X: K$ is compact subset of $X\}$. Suppose the following conditions are fulfilled:

(i) $f(x, y) \leq g(x, y)$ for all $(x, y) \in X \times Y$,

(ii) $f(x, y)$ is lower semicontinuous on $X$ for each $y \in Y$,

(iii) $g(x, y)$ is $H$-quasiconcave on $Y$ for each $x \in X$,

(iv) $g(x, y)$ is lower semicontinuous on $X \times Y$ and the set $\{x \in K: g(x, y)<t\}$ is acyclic or empty for each $t>\beta, K \in \bar{K}$ and $y \in Y$. Then

$$
\inf _{x \in X} \sup _{y \in Y} f(x, y) \leq \inf _{K \in \bar{K}} \sup _{y \in Y} \min _{x \in K} g(x, y) .
$$

If $X$ is compact, then

$$
\min _{x \in X} \sup _{y \in Y} f(x, y) \leq \sup _{y \in Y} \min _{x \in X} g(x, y) .
$$

Corollary 2.3. Let $f: X \times Y \rightarrow R$ be a real-valued function. Let $\beta=$ $\inf _{K \in \bar{K}} \sup _{y \in Y} \min _{x \in K} f(x, y)$, where $\bar{K}=\{K \subset X: K$ is compact subset of $X\}$. Suppose the following conditions are fulfilled:

(i) $f(x, y)$ is lower semicontinuous on $X \times Y$,

(ii) $f(x, y)$ is $H$-quasiconcave on $Y$ for each $x \in X$, and the set $\{x \in K$ : $f(x, y)<t\}$ is acyclic or empty for each $t>\beta, K \subset \bar{K}$ and $y \in Y$. Then

$$
\inf _{x \in X} \sup _{y \in Y} f(x, y)=\inf _{K \in \bar{K}} \sup _{y \in Y} \min _{x \in K} f(x, y) .
$$


If $X$ is compact, then

$$
\min _{x \in X} \sup _{y \in Y} f(x, y)=\sup _{y \in Y} \min _{x \in X} f(x, y) .
$$

Remark 2.3. Corollary 2.3 is the generalization of [1, Theorem 4], thus, Theorem 2.2, Corollary 2.1 and Corollary 2.2 are all the generalizations of [1, Theorem 4].

Theorem 2.4. Let $Y$ be a compact Hausdorff topological space and $\left(X,\left\{\Gamma_{A}\right\}\right)$ be an $H$-space, let $f, g: X \times Y \rightarrow R$ be two real-valued functions such that:

(i) $f(x, y) \leq g(x, y)$ for all $(x, y) \in X \times Y$,

(ii) $f(x, y)$ is $H$-quasiconvex on $X$ for each $y \in Y$,

(iii) $g(x, y)$ is upper semicontinuous on $Y$ for each $x \in X$.

If $T$ is an upper semicontinuous set-valued mapping defined on $X$ such that $T x$ is a nonempty compact acyclic subset of $Y$ for each $x \in X$, then

$$
\inf _{y \in T x} f(x, y) \leq \max _{y \in Y} \inf _{x \in X} g(x, y) .
$$

Proof. If the conclusion of Theorem 2.4 is false, then there is a real number $t$ such that

$$
\inf _{y \in T x} f(x, y)>t>\max _{y \in Y} \inf _{x \in X} g(x, y) .
$$

Let

$$
M=\{(x, y) \in X \times Y: f(x, y) \geq t\}, P=\{(x, y) \in X \times Y: y \in T x\},
$$

and $Y_{x}=\{y \in Y: g(x, y) \geq t\}$ for each $x \in X$. It is easy to verify that $M$ and $Y_{x}$ satisfies (i) and (ii) of Theorem 2.1, and $P$ is closed in $X \times Y$ and satisfies (iii) of Theorem 2.1 by taking $K=Y$. Thus, by theorem $2.1, \bigcap_{x \in X} Y_{x} \cap Y \neq \emptyset$, that is, there exists $y_{0} \in Y$ such that

$$
g\left(x, y_{0}\right) \geq t, \text { for all } x \in X .
$$

Hence

$$
\max _{y \in Y} \inf _{x \in X} g(x, y) \geq t
$$

This contradicts the choice of $t$, therefore, (2.5) is proved.

Remark 2.4. Theorem 2.4 is the generalization of [2, Theorem 1].

By Theorem 2.4, we can obtain the following corollary. 
Corollary 2.4. Let $f, g, T$ be as in Theorem 2.4. Assume further, that given $\lambda \in R$, we have

$$
\inf _{y \in T x} f(x, y) \geq \lambda \text { for all } x \in X .
$$

Then there exists $y_{0} \in Y$ such that $g\left(x, y_{0}\right) \geq \lambda$ for all $x \in X$.

Remark 2.5. Corollary 2.4 is similar to the result of [4, Theorem 13.4].

We can obtain the following two theorems whose proofs are similar to the proof of Theorem 2.4.

Theorem 2.5. Let $\left(Y,\left\{\Gamma_{A}\right\}\right)$ be a Hausdorff $H$-space, let $X$ be nonempty compact acyclic $H$-convex subset of $Y$. Let $f, g: X \times Y \rightarrow R$ be two real-valued functions satisfying $(i)-($ iii) of Theorem 2.4. Then

$$
\inf _{x \in X} f(x, x) \leq \sup _{y \in Y} \inf _{x \in X} g(x, y) .
$$

Theorem 2.6. Let $\left(Y,\left\{\Gamma_{A}\right\}\right)$ be a Hausdorff $H$-space, let $X$ be nonempty compact acyclic $H$-convex subset of $Y$. Let $f: X \times Y \rightarrow R$ be a real-valued function such that

(i) $f(x, y)$ is a $H$-quasiconvex on $X$ for each $y \in Y$,

(ii) $f(x, y)$ is upper semicontinuous on $Y$ for each $x \in X$.

Then

$$
\inf _{x \in X} f(x, x) \leq \sup _{y \in Y} \inf _{x \in X} f(x, y) .
$$

Corollary 2.5. Let $\left(Y,\left\{\Gamma_{A}\right\}\right)$ be a Hausdorff $H$-space, let $X$ be nonempty compact acyclic $H$-convex subset of $Y$. Let $f, g: X \times Y \rightarrow R$ be two real-valued functions such that

(i) $f(x, y) \leq g(x, y)$ for all $(x, y) \in X \times Y$,

(ii) $f(x, y)$ is lower semicontinuous on $Y$ for each $x \in X$,

(iii) $g(x, y)$ is $H$-quasiconcave on $X$ for each $y \in Y$. Then

$$
\inf _{y \in Y} \sup _{x \in X} f(x, y) \leq \sup _{x \in X} g(x, x) .
$$

\section{ACKNOWLEDGMENTS}

The authors truly appreciates the helpful remarks and valuable suggestions of the anonymous referees, which improves this paper. 


\section{REFERENCES}

1. C. W. Ha, Minimax and fixed point theorems, Math. Ann. 248 (1980), 73-77.

2. C. W. Ha, On a minimax inequality of Ky Fan, Proc. Amer. Math. Soc. 99 (1987), 680-682.

3. K. Fan, Some properties of convex sets relation to fixed point theorems, Math. Ann. 266 (1984), 519-537.

4. A. Granas and F. C. Liu, Coincidence for set-valued maps and minimax inequalities, J. Math. Pure. Appl. 65 (1986), 119-148.

5. K. K. Tan, Comparison theorems on minimax inequalities, variational inequalities, $J$. London. Math. Soc. 28 (1983), 555-562.

6. S. Kum, A generalization of generalized quasivariational inequalities, J. Math. Anal. Appl. 182 (1994), 158-164.

7. J. Y. Fu, Implict variational inequalities for multivalued mappings, J. Math. Anal. Appl. 189 (1995), 801-814.

8. K. Fan, A generalization of Tychonoff's fixed theorem, Math. Ann. 142 (1961), 305-310.

9. C. Horvath, Point fixes et coincidences pour les applicatios multivoques sans convexite, C. R. Acad. Sci. Paris. 296 (1983), 403-406.

10. C. Horvath, Point fixes et coincidences dans les espaces topologiques compacts contractiles, C. R. Acad. Sci. Paris. 299 (1984), 519-521.

11. C. Horvath, Some results on multivalued mappings and inequalities without convexity, in "Nonlinear and Convex Analysis" (B. L. Lin and S. Simons, Eds.), Marcel Dekker. New York, 1987, pp. 99-106.

12. E. Tarafdar, Fixed point theorems in $H$-spaces and equilibrium points of abstract economies, J. Austral. Math. Soc. 53 (1992), 252-260.

13. S. Park, J. S. Bae and H. K. Kang, Geometric properties minimax inequalities, and fixed point theorems on convex spaces, Proc. Amer. Math. Soc. 121 (1994), 429-439.

14. C. Berge, Topological Spaces, Oliver and Boyd, Edinburgh, London, 1963.

15. X. Wu and F. Li, On Ky Fan's Section Theorem, J. Math. Anal. Appl. 227 (1998), $112-121$.

16. J. H. Zhang, Minimax inequalities of Ky Fan, Appl. Math. Lett. 11 (1998), 37-41.

17. J. F. McClendon, Minimax and variational inequalities for compact spaces, Proc. Amer. Math. Soc. 89 717-721, (1983). 
Lu Haishu,

College of Water Conservancy and Hydropower Engineering,

Hohai University,

Nanjing 210098, Jiangsu,

P. R. China

Zhang Jihui,

Department of Mathematics,

Nanjing Normal University,

Nanjing 210097, Jiangsu,

P. R. China.

E-mail: jihuiz@jlonline.com 